\title{
Les pouvoirs publics et les édifices cultuels en Belgique
}

\section{Jean-François Husson}

\section{OpenEdition}

\section{Journals}

Édition électronique

URL : http://journals.openedition.org/rdr/831

DOI : $10.4000 /$ rdr.831

ISSN : 2534-7462

\section{Éditeur}

Presses universitaires de Strasbourg

Édition imprimée

Date de publication : 3 mai 2017

Pagination : 61-78

ISBN : 978-2-86820-973-3

ISSN : 2493-8637

\section{Référence électronique}

Jean-Francois Husson, «Les pouvoirs publics et les édifices cultuels en Belgique », Revue du droit des religions [En ligne], 3 | 2017, mis en ligne le 03 février 2020, consulté le 19 novembre 2020. URL : http:// journals.openedition.org/rdr/831 ; DOI : https://doi.org/10.4000/rdr.831

\section{(c) (1) 8)}

La revue du droit des religions est mise à disposition selon les termes de la Creative Commons Attribution - Pas d'Utilisation Commerciale 4.0 International - CC BY-NC 4.0. 


\section{LES POUVOIRS PUBLICS ET LES ÉDIFICES CULTUELS EN BELGIOUE}

\section{Jean-François HUSSON}

Observatoire des relations administratives entre les cultes, la laïcité organisée et l'État (ORACLE) et Université de Liège

\section{RÉSUMÉ}

Le régime belge de relations entre l'État et les communautés religieuses et philosophiques reconnues se traduit notamment par des soutiens financiers aux bâtiments affectés au culte ou à l'assistance morale. Ces interventions s'inscrivent pour l'essentiel dans un dispositif hérité de l'époque concordataire française, peu remis en cause par la large régionalisation de la matière. Ce dispositif est aujourd'hui sous tension, car il doit répondre à des situations contrastées, entre cultes reconnus au XIX ${ }^{e}$ siècle - généralement en déclin - et cultes reconnus plus récemment - généralement en expansion. La situation est également complexifiée par des différences liées à la propriété des bâtiments ou à leur classement au titre du patrimoine culturel. La présente contribution questionne l'équité du dispositif et aborde les développements possibles.

\section{ABSTRACT}

The Belgian regime of relations between the State and religious or philosophical communities results notably in financial support for buildings used for worship and moral counselling. Those interventions are essentially a legacy of the French Concordat, largely unchallenged by the regionalization process. Today, it has to respond to contrasting situations between religions recognized in the 19th century - generally declining - and more recently recognized ones - generally expanding. An additional complication originates in differences in ownership of the buildings or their classification as listed buildings. After presenting the situation by religious and philosophical community and level of power, this paper questions the equity of the system and addresses the possible developments. 
T es cultes et organisations philosophiques non confessionnelles présents Len Belgique connaissent des situations contrastées quant à leurs lieux de culte ou autres implantations. La présente contribution propose d'en présenter le cadre global ainsi que ses implications quant à la gestion des édifices du culte, classés ou non.

\section{LE DISPOSITIF EXISTANT : LE POIDS DU PASSÉ}

La Belgique connaît un régime de cultes reconnus ${ }^{1}$, élargi à la fin du $\mathrm{xx}^{\mathrm{e}}$ siècle aux « organisations reconnues par la loi qui offrent une assistance morale selon une conception philosophique non confessionnelle $»^{2}$.

Une reconnaissance s'accompagne d'un soutien financier, reposant principalement sur des instruments instaurés par le Concordat français de 1801 et des législations subséquentes ${ }^{3}$. Ce dispositif, fondé sur la civilisation paroissiale, obéit au schéma suivant, initialement prévu pour le culte catholique : les fidèles se retrouvent au lieu de culte (dont l'entretien et le coût de fonctionnement sont supportés par la commune en cas d'insuffisance des revenus de la fabrique) sous la guidance du ministre du culte (payé par l'État). Cette configuration a été conservée jusqu'à ce jour ${ }^{4}$. Dans ce cadre, la gestion des lieux de culte incombe alors à un organe qui a le statut d'établissement public, tels les fabriques d'église catholiques ou les comités islamiques.

La gestion des lieux de culte réside principalement entre les mains de deux types d'organes de gestion ${ }^{5}$ :

1. Les cultes catholique, protestant et israélite (reconnus dès l'indépendance), anglican (reconnu au XIX ${ }^{\mathrm{e}}$ s.), islamique (1974) et orthodoxe (1985).

2. Selon les termes de l'art. 181 de la Constitution. Actuellement, il s'agit uniquement de la « laïcité organisée », c'est-à-dire la communauté libre-exaministe, mais le bouddhisme a demandé à être reconnu comme tel et non comme un culte.

3. Les territoires de l'actuelle Belgique (principalement les Pays-Bas autrichiens et la Principauté de Liège) avaient été "réunis » à la France par le décret du 9 vendémiaire an IV ( $1^{\text {er }}$ oct. 1795) qui établit la réunion de la Belgique et du pays de Liège à la France.

4. Le décret impérial du 30 décembre 1809 sur les fabriques des églises, modifié, est d'ailleurs toujours d'application dans les Régions wallonne et de Bruxelles-Capitale tandis que les dispositions décrétales adoptées par la Flandre et la Communauté germanophone en reprennent l'esprit, voire certaines dispositions.

5. Davagle M. et Husson J.-F., « Brève comparaison entre le fonctionnement des établissements chargés de la gestion du temporel du culte et celui des ASBL », Les Dossiers d'ASBL Actualités, $\mathrm{n}^{\circ} 13$, Les fabriques d'église, 2012, p. 83-115. 
- les fabriques d'église - et leurs équivalents au sein des autres cultes qui sont des établissements publics faisant l'objet d'une reconnaissance officielle et soumis à un ensemble de dispositions légales et réglementaires en matière de fonctionnement, de tutelle et de financement; ce statut sera donc utilisé pour les communautés cultuelles ou philosophiques reconnues ;

- les associations sans but lucratif (ASBL), personnes morales de droit privé, soumises à une réglementation générale ${ }^{6}$ mais ne requérant pas de reconnaissance officielle et jouissant d'une large autonomie, dans le cadre des statuts dont elles se dotent. Ces ASBL correspondent, pour l'essentiel, aux associations loi 1901 françaises, la législation belge n'ayant pas de statut particulier pour les associations cultuelles ; ce statut est utilisé par les communautés non reconnues (le cas échéant, préalablement à leur reconnaissance) ainsi que pour les activités périphériques, en particulier dans les champs socioculturel et caritatif.

Enfin, à la suite de la réforme de l'État de $2001^{7}$, les compétences autrefois fédérales sont dorénavant réparties comme suit ${ }^{8}$ :

- État fédéral : reconnaissance des cultes et des organisations philosophiques non confessionnelles ainsi que de leurs organes représentatifs, prise en charge des traitements et pensions des ministres des cultes et délégués des organisations philosophiques; législation et tutelle relatives aux établissements des organisations philosophiques;

- Régions ${ }^{9}$ : législation et tutelle relatives aux établissements cultuels locaux (y compris critères et procédure de reconnaissance); travaux sur les édifices des cultes et le patrimoine protégé ${ }^{10}$;

6. V. loi du 27 juin 1921 sur les associations sans but lucratif, les associations internationales sans but lucratif et les fondations.

7. Loi spéciale du 13 juillet 2001 portant transfert de diverses compétences aux Régions et Communautés.

8. Les entités fédérées, Communautés et Régions, peuvent être visualisées sur cette page http:// www.belgium.be/fr/la_belgique/pouvoirs_publics/la_belgique_federale/Carte [consulté le 13 janv. 2017].

9. Ainsi que la Communauté germanophone en application du décret du 27 mai 2004 relatif à l'exercice, par la Communauté germanophone, de certaines compétences de la Région wallonne en matière de pouvoirs subordonnés.

10. «Par patrimoine, il faut entendre l'ensemble des biens immobiliers dont la protection se justifie en raison de leur intérêt historique, archéologique, architectural, scientifique, artistique, social, mémoriel, esthétique, technique, paysager ou urbanistique, en tenant compte des critères soit de rareté, soit d'authenticité, soit d'intégrité, soit de représentativité " (extrait de l'art. 185 du Code wallon de l'aménagement du territoire, de l'urbanisme, du patrimoine et de l'énergie). 
- Communautés ${ }^{11}$ : patrimoine mobilier (dont les archives et certaines œuvres d'art) ${ }^{12}$.

$\mathrm{Au}$ lieu d'un régime unique pour l'ensemble du pays, coexistent actuellement quatre cadres légaux différents pour les cultes (Régions flamande, wallonne et de Bruxelles-Capitale ; Communauté germanophone) ainsi qu'un cadre légal pour les organisations philosophiques non confessionnelles (Autorité fédérale). Des différences existent ainsi entre entités fédérées mais également, sur un même territoire, entre les cultes relevant de la législation fédérée et les organisations philosophiques relevant de la législation fédérale.

\section{LA SITUATION PATRIMONIALE DES PRINCIPALES COMMUNAUTÉS CONVICTIONNELLES : UN PAYSAGE CONTRASTÉ}

La situation est contrastée et une distinction peut être opérée entre les cultes reconnus au XIX ${ }^{e}$ siècle (cultes catholique, protestant $[\mathrm{EPUB}]^{13}$, israélite et anglican) et ceux reconnus aux $\mathrm{xx}^{\mathrm{e}}$ et $\mathrm{xxI}^{\mathrm{e}}$ siècles (cultes islamique et orthodoxe ainsi que les évangéliques). Les premiers ont des effectifs au mieux stables, plus généralement en déclin; leurs racines historiques expliquent qu'une part plus ou moins importante de leurs lieux de culte est classée et peut bénéficier des interventions au titre du patrimoine. Les seconds, dont les effectifs sont en croissance, n'ont pas le même enracinement historique et ne bénéficient que rarement des interventions au titre du patrimoine. La situation de la « laïcité organisée » est, quant à elle, spécifique.

11. Nous utilisons « Communauté » pour les institutions politiques et « communauté » pour les communautés cultuelles ou philosophiques.

12. «Le décret du 11 juillet 2002 relatif aux biens culturels mobiliers et au patrimoine immatériel de la Communauté française permet la protection des biens culturels les plus importants et les plus significatifs situés en Fédération Wallonie-Bruxelles [...]. La protection des biens culturels mobiliers touche un large éventail d'objets, allant des œuvres d'art aux objets archéologiques, aux moyens de transport sans oublier les documents d'archives, les pièces ethnographiques, les objets d'art décoratifs, les instruments scientifiques. [...]»: http://www.patrimoineculturel.cfwb.be/index.php?id=7244 [consulté le 13 janv. 2017].

13. La Belgique reconnaît aujourd'hui le culte protestant-évangélique, composé de l'Église protestante unie de Belgique (EPUB), représentant le protestantisme « historique » et certains de ses partenaires (tels les adventistes), et le Synode fédéral des Églises évangéliques de Belgique. Les deux courants ayant connu des développements différents, ils seront distingués dans notre propos. 


\subsection{LE PATRIMOINE DES CULTES RECONNUS AU XIXE SIÈCLE}

Pays fortement marqué par le catholicisme ${ }^{14}$, la Belgique compte de nombreux lieux de culte catholiques, dont une proportion substantielle est classée. Les pouvoirs publics sont propriétaires d'une part importante des lieux de culte, principalement des bâtiments antérieurs à 1795; les autres appartiennent aux fabriques et, pour le solde, à des congrégations ou autres propriétaires privés. Les presbytères appartiennent tantôt aux pouvoirs publics, tantôt aux fabriques. Ils sont de moins en moins occupés et la pression des pouvoirs locaux pour leur désaffectation est forte; nombre d'entre eux ont ainsi été réaffectés en crèches, pour l'accueil des réfugiés, etc. La plupart des fabriques d'église disposent de biens immobiliers non affectés au culte : terrains agricoles, immeubles de rapport, logements sociaux... ${ }^{15}$. Les recettes qui en découlent réduisent le déficit à la charge des pouvoirs publics. Malgré certaines pressions émanant principalement d'élus locaux, il n'est toutefois pas dans la logique actuelle des autorités de tutelle de permettre la vente de tels biens afin de financer des gros travaux sur les lieux de culte. Relevons également que des interventions publiques visant à améliorer les biens de rapport sont possibles mais facultatives; pour les pouvoirs publics, il peut s'agir d'une sorte d'investissement (l'amélioration du rendement locatif peut améliorer les recettes de la fabrique et réduire de ce fait l'intervention publique) ou d'un choix politique, par exemple en faveur du logement social.

Le culte catholique est confronté à une situation difficile, car ce grand nombre d'églises et de chapelles est desservi par des effectifs limités et vieillissants. Les coûts d'entretien, de rénovation et de fonctionnement s'avèrent relativement élevés alors que les pouvoirs locaux - soumis à d'importantes contraintes budgétaires - rechignent de plus en plus à les supporter; quant à la pratique religieuse, elle poursuit sa baisse tendancielle ${ }^{16}$. Si les autorités

14. Le recensement de 1846 comptait plus de $99 \%$ de catholiques (Rigaux M.-F. et al., Le financement par l'État fédéral des ministres des cultes et des délégués du Conseil central laïque. Rapport de la Commission des sages, Bruxelles, SPF Justice, 2006), le protestantisme ayant été quasiment éradiqué aux $\mathrm{XVI}^{\mathrm{e}}$ et $\mathrm{XVII}^{\mathrm{e}}$ siècles avant de connaître diverses phases de développement à partir de la fin du $\mathrm{XIX}^{\mathrm{e}}$ siècle.

15. Les bâtiments affectés à la vie socio-culturelle locale (mouvements de jeunesse, salles paroissiales, etc.) appartiennent généralement à des associations gravitant autour de la paroisse, en particulier les associations d'œuvres paroissiales, sous le statut d'ASBL expliqué plus loin.

16. ARCQ É. et SÄGESSER C., «Le fonctionnement de l'Église catholique dans un contexte de crise ", Courrier hebdomadaire du Centre de recherche et d'information socio-politiques, $\mathrm{n}^{\circ}$ 2112-2113, 2011, p. 3-85. 
religieuses sont - dans certains diocèses - disposées à "rationaliser » ce parc immobilier en envisageant la désaffectation de certains lieux de culte, les fidèles - voire la population locale - peuvent se révéler extrêmement réticents ${ }^{17}$. À cela s'ajoutent des lieux de culte classés au patrimoine exceptionnel, voire au patrimoine mondial ${ }^{18}$, qui bénéficient d'importantes interventions publiques.

L'Église protestante unie de Belgique (EPUB) dispose d'une centaine de communautés, qui se maintiennent mais ne se développent pas. Les temples sont le plus souvent la propriété d'ASBL locales ou nationales (par ex. la Mission méthodiste). Une exception notable est la chapelle royale protestante, qui jouxte le musée des Beaux-Arts à Bruxelles et est propriété de la Régie des bâtiments (État fédéral). Un certain nombre de temples sont classés ${ }^{19}$.

Le culte israélite dispose d'une dizaine de communautés. Certaines synagogues ont été construites avant la Première Guerre mondiale ${ }^{20}$ et sont généralement classées. Aucune n'appartient aux pouvoirs publics.

Le culte anglican dispose de lieux de culte appartenant à la fabrique d'église tandis que d'autres sont propriétés de la municipalité dans laquelle ils sont situés ou de son centre public d'action sociale. D'autres, enfin, appartiennent à des personnes morales de droit privé ou à des congrégations religieuses ${ }^{21}$.

Si le culte catholique sollicite régulièrement les pouvoirs publics pour des travaux sur ses lieux de culte, les demandes émanant des communautés protestantes et israélites sont plus rares et souvent limitées à des situations particulières (bâtiments classés et/ou appartenant à l'État).

\subsection{LE PATRIMOINE DES CULTES RECONNUS DEPUIS $1970^{22}$}

Ces cultes - islamique, orthodoxe et évangélique - sont reconnus, mais certaines communautés locales ne sont pas reconnues pour diverses raisons

17. Le cas des églises Sainte-Catherine à Bruxelles ou Saint-Jacques à Namur sont deux exemples particulièrement illustratifs.

18. Telle la cathédrale de Tournai.

19. De PoOter P., De rechtspositie van erkende erediensten en levensbeschouwingen in Staat en maatschappij, Brussel, Larcier, 2003.

20. Signalons que la plupart d'entre elles furent construites dans un style oriental alors que la plupart des juifs belges étaient originaires d'Europe centrale. La conception de l'altérité à l'époque était apparemment quelque peu différente de ce qu'elle est aujourd'hui.

21. De Pooter P., op. cit.

22. Le cas des cultes non reconnus ne sera pas abordé. Signalons simplement qu'ils peuvent se constituer en ASBL et bénéficier de l'exonération d'impôt foncier, ainsi que des subsides pour le patrimoine classé s'ils occupent de tels bâtiments. 
(demande n'ayant pas encore abouti, demande non encore introduite, souhait de ne pas être reconnu compte tenu des contraintes administratives en découlant).

Pour acquérir ou louer des bâtiments destinés au culte, ces communautés se sont généralement constituées en associations sans but lucratif (ASBL). Lorsqu'une communauté locale est reconnue par les pouvoirs publics, l'ASBL peut transférer la propriété du bien à l'établissement public constitué à la suite de la reconnaissance. Cependant, dans de nombreux cas, les acteurs locaux ont préféré conserver la propriété au sein de l'ASBL et mettre le bâtiment à disposition, à titre gracieux, via une location ou encore via un bail emphytéotique.

$\mathrm{Au}$ sein du culte islamique, les bâtiments appartiennent ainsi aux ASBL locales ou à des fédérations. Dans le cas des mosquées turques affiliées à la Diyanet de Belgique ${ }^{23}$, financées à l'origine par les fidèles, la propriété est transférée à la Diyanet qui, en contrepartie, apporte divers soutiens à la communauté locale et lui fournit un imam, dont la présidence des Affaires religieuses turque supporte la charge. Une mosquée appartient toutefois à l'État belge : il s'agit de la Grande Mosquée de Bruxelles, aussi appelée mosquée du Cinquantenaire. Celle-ci est tout à fait particulière : aujourd'hui intégrée au réseau de la Ligue islamique mondiale et présidée par l'ambassadeur d'Arabie saoudite, elle a fait l'objet d'un bail emphytéotique lorsque les autorités belges ont souhaité lui confier le rôle de représentation de l'islam en Belgique. Ce rôle fut contesté par diverses parties et lui fut graduellement retiré. Compte tenu de son positionnement et de ses affiliations, la Grande Mosquée n'est pas reconnue par la Région de Bruxelles-Capitale (elle n'a apparemment jamais introduit de demande) et n'a pas participé au dernier processus de renouvellement de l'Exécutif des musulmans de Belgique, organe représentatif reconnu, en 2013-2014. Cela peut apparaître étonnant s'agissant de la seule mosquée occupant un bâtiment public. Actuellement, près de 80 mosquées sont reconnues sur un total estimé à environ 300 mosquées. Une spécificité du culte islamique est qu'un même bâtiment abrite non seulement le lieu de culte mais aussi des espaces socioculturels (cours de langue, cours sur le Coran, etc.). Cela ne va pas sans poser diverses questions pratiques dans le cas des mosquées reconnues; les interventions publiques (couverture du déficit, exonération d'impôt foncier) n'étant pas identiques pour les activités cultuelles et les autres activités. Cela pose également le problème,

23. La Diyanet de Belgique, branche belge de la présidence des Affaires religieuses turque, est constituée sous forme d'association internationale de droit belge. 
parfois aigu, de la cohabitation de deux organes de gestion, l'un pour l'établissement public (comité islamique de la mosquée reconnue), l'autre pour l'ASBL. Leurs modes d'élection étant différents (ensemble des fidèles pour le premier, membres pour la seconde), des tensions sont parfois apparues, y compris à propos des activités organisées. Au cours des dernières années, un certain nombre de mosquées ont fait l'objet d'aménagements importants ou de constructions neuves; cela n'a pas toujours été sans difficulté en matière de respect des règles d'urbanisme, d'acceptabilité de certaines caractéristiques architecturales (à commencer par les minarets, souvent évités de ce fait) par les administrations concernées et les riverains ou des inconvénients, avérés ou supposés, découlant de l'implantation d'un lieu de culte fréquenté (en particulier en matière de circulation et de parking). Enfin, l'origine des fonds destinés à certains chantiers a suscité des controverses.

Le culte orthodoxe est composé de diverses Églises nationales ainsi que de communautés relevant du Patriarcat œecuménique. Les communautés locales se sont constituées en ASBL, bénéficiant le cas échéant du soutien des pays d'origine. Lorsque des communautés ont été reconnues, le lieu de culte est généralement demeuré dans le giron de l'ASBL, un contrat de location étant conclu avec l'établissement public.

Le Synode fédéral des Églises évangéliques regroupe de très nombreuses confessions, ayant elles-mêmes un nombre variable de communautés locales. Le nombre de communautés reconnues est limité. Il existe également des communautés évangéliques n'ayant pas intégré le Synode fédéral. Dans les deux cas, les bâtiments appartiennent à des ASBL, mais de nombreuses communautés recourent à la location. Des travaux sur l'implantation des communautés évangéliques à Bruxelles ont ainsi montré une très grande mobilité de celles-ci sur le territoire de la Région de Bruxelles-Capitale ${ }^{24}$, ce qui constitue un phénomène nouveau. Cette mobilité résulte de la recherche de bâtiments plus adaptés compte tenu de l'évolution des effectifs de la communauté concernée, voire - parfois - des dispositions liées à la sécurité et à la salubrité des bâtiments.

Un point commun à ces cultes, en particulier islamique et évangélique, est le souhait de jouir de lieux de culte supplémentaires ou davantage adaptés en taille et/ou en aménagements à leurs activités.

24. Menier B., "Les lieux de culte évangéliques à Bruxelles », 28 avril 2012, en ligne sur ORELA : http://www.o-re-la.org [consulté le 13 janv. 2017]. 


\subsection{LA « LAÏCITÉ ORGANISÉE » ${ }^{25}$}

Le mouvement laïque s'est graduellement structuré au fil du temps, avec des ASBL tant sur une base locale (dont les Maisons de la laïcité) que sectorielle. Ces ASBL étaient elles-mêmes fédérées dans des ASBL faîtières qui, à leur tour, ont constitué le Centre d'action laïque francophone et son pendant néerlandophone, formant ensemble le Conseil central laïque. La reconnaissance de ce mouvement s'est opérée en plusieurs étapes : octroi d'un subside destiné à faciliter la structuration du mouvement à partir de 1980, modification de la Constitution en 1993 et, in fine, la loi du 21 juin 2002 relative au Conseil central des communautés philosophiques non confessionnelles de Belgique, aux délégués et aux établissements chargés de la gestion des intérêts matériels et financiers des communautés philosophiques non confessionnelles reconnues. Il en découle une double structure constituée d'ASBL d'une part et des établissements publics mis en place dans le cadre de la loi de 2002 d'autre part ${ }^{26}$.

Les Maisons de la laïcité ${ }^{27}$ ont pu disposer de bâtiments publics (parfois d'anciennes mairies, situées à proximité de l'église locale, ce qui n'est pas sans portée symbolique) et/ou d'aides publiques à l'achat et à l'aménagement des bâtiments. Ces interventions peuvent apparaître comme un phénomène de « rattrapage ${ }^{28}$ par rapport à la présence catholique; elles ont par la suite été étendues aux bâtiments des "établissements publics d'assistance morale ${ }^{29}$, implantations décentralisées du Conseil central laïque.

\section{LES INSTRUMENTS : DU NEUF AVEC DU VIEUX?}

Face à ces besoins et développements parfois contradictoires, les instruments publics de régulation et de soutien financier - sur lesquels nous nous concentrerons - sont toujours ceux mis en place au début du XIX siècle pour

25. Pour une contextualisation: Husson J.-F., « La libre-pensée, une religion comme une autre? Financement public et gestion de la pluralité religieuse et philosophique en Belgique », in Lamine A.-S., Quand le religieux fait conflit. Désaccords, négociations ou arrangements, Presses universitaires de Rennes, 2014, p. 47-60.

26. Husson J.-F. et SÄgesSER C., «La reconnaissance et le financement de la laïcité (II)», Courrier hebdomadaire du CRISP, $\mathrm{n}^{\circ} 1760,2002$, p. 3-52.

27. Il s'agit d'ASBL et non d'établissements publics. V. les documents cités dans les notes 28 et 29 pour une explication détaillée.

28. Husson J.-F., "Le financement des cultes, de la laïcité et des cours philosophiques», Courrier hebdomadaire du CRISP, $\mathrm{n}^{\circ}$ 1703-1704, 2000, p. 3-90.

29. Jeholet P.-Y., SÄGesser C. et Husson J.-F., La législation wallonne sur les cultes : état des lieux et pistes de réformes, Namur, Parlement wallon, 2014. 
le culte catholique. À cet égard, le dispositif belge a pu intégrer l'évolution de la diversité religieuse et philosophique, bien que la répartition des moyens reçus par les diverses communautés convictionnelles puisse interpeller. Les principales interventions publiques au titre des cultes, déjà citées, peuvent être précisées comme suit.

Tableau 1. Principales interventions publiques ${ }^{30}$

\begin{tabular}{|c|c|c|c|}
\hline $\begin{array}{c}\text { Nature } \\
\text { de la dépense }\end{array}$ & $\begin{array}{l}\text { Niveau } \\
\text { de pouvoir } \\
\text { établissant } \\
\text { le cadre légal/ } \\
\text { réglementaire }\end{array}$ & $\begin{array}{l}\text { Nature } \\
\text { de la dépense } \\
\text { (pour les } \\
\text { établissements } \\
\text { reconnus) }\end{array}$ & $\begin{array}{c}\text { Niveau } \\
\text { de pouvoir } \\
\text { supportant } \\
\text { la dépense }(a)\end{array}$ \\
\hline $\begin{array}{l}\text { Frais } \\
\text { de fonctionnement } \\
\text { (chauffage, eau, } \\
\text { assurances, } \\
\text { entretien courant...) }\end{array}$ & Régions (b) (c) & $\begin{array}{l}\text { Couverture } \\
\text { du déficit } \\
\text { de l'établissement } \\
\text { (obligatoire) }\end{array}$ & $\begin{array}{l}\text { Communes } \\
\text { ou provinces } \\
\text { selon le culte } \\
\text { (service ordinaire } \\
\text { du budget) }\end{array}$ \\
\hline $\begin{array}{l}\text { Gros entretien, } \\
\text { réparations, } \\
\text { reconstruction }\end{array}$ & Régions (b) (c) & Obligatoire & $\begin{array}{l}\text { Communes } \\
\text { ou provinces selon } \\
\text { le culte (service } \\
\text { extraordinaire } \\
\text { du budget). Subsides } \\
\text { régionaux possibles }\end{array}$ \\
\hline Construction & Régions (b) (c) & Facultative & $\begin{array}{l}\text { Communes } \\
\text { ou provinces selon } \\
\text { le culte (service } \\
\text { extraordinaire } \\
\text { du budget). Subsides } \\
\text { régionaux possibles }\end{array}$ \\
\hline $\begin{array}{l}\text { Fiscalité } \\
\text { immobilière (d) }\end{array}$ & Régions & $\begin{array}{l}\text { Exonération } \\
\text { du précompte } \\
\text { immobilier } \\
\text { (impôt foncier) }\end{array}$ & $\begin{array}{l}\text { Dépense fiscale } \\
\text { pour les communes, } \\
\text { les provinces, } \\
\text { les Régions }\end{array}$ \\
\hline
\end{tabular}

30. Pour une information détaillée concernant les montants mobilisés, V. Husson J.-F., « Le financement des cultes, de la laïcité et des cours philosophiques », art. cit. ; Husson J.-F. (éd.), Le financement des cultes et de la laïcité. Comparaison internationale et perspectives, Namur, Éd. namuroises, 2005 ; Jeholet P.-Y, SÄGESSER C. et HusSOn J.-F., op. cit. 
(a) Communes pour les cultes catholique (hors fabriques cathédrales), protestant-évangélique, israélite et anglican. Provinces (ou Région de Bruxelles-Capitale sur son territoire) pour les cultes catholique (fabriques cathédrales), islamique et orthodoxe ainsi que pour les établissements d'assistance morale non confessionnelle.

(b) Communauté germanophone sur son territoire.

(c) État fédéral pour les organisations philosophiques non confessionnelles.

(d) Non limité aux seuls cultes et organisations philosophiques reconnus.

En matière de cultes et d'assistance philosophique, l'intervention des pouvoirs publics est supplétive, qu'il s'agisse du fonctionnement ou des investissements. La part incombant à la personne morale gérant le lieu de culte peut être partiellement couverte par des recettes provenant de collectes, de dons, de revenus immobiliers ou de diverses activités, ces dernières étant généralement organisées par des ASBL ${ }^{31}$. Les subsides au titre des cultes sont tributaires de plusieurs conditions, la principale étant que le bâtiment appartienne à un pouvoir ou établissement public ou qu'il fasse l'objet d'un bail emphytéotique ; un bâtiment loué sur la base d'un bail commun ne peut en bénéficier.

À cela s'ajoutent des subventions déliées de l'affectation cultuelle du bâtiment :

- subventions régionales au titre de la préservation du patrimoine historique ou architectural, lesquelles représentent des montants considérables en Région flamande;

- financements fédéraux pour la promotion du rôle fédéral et international de Bruxelles (en Région de Bruxelles-Capitale);

- subventions des Communautés pour le patrimoine mobilier;

- subventions régionales au titre de l'environnement, des économies d'énergie ou du tourisme ;

- prise en charge directe de travaux et autres coûts relatifs à des bâtiments appartenant ou confiés aux pouvoirs publics (par exemple la Régie des bâtiments de l'État fédéral ou l'Institut du patrimoine wallon).

Dans tous les cas, les pouvoirs locaux peuvent également décider d'octroyer des subventions facultatives, discrétionnaires, lesquelles viennent le cas échéant s'ajouter aux interventions obligatoires. Enfin, concernant les bâtiments classés (avec différents niveaux de classement ${ }^{32}$ ), l'Institut du patrimoine wallon, tout

31. Dans certains cas, les dons peuvent bénéficier d'une déductibilité fiscale dans le chef des donateurs.

32. V. Code wallon de l'aménagement du territoire (art. 187 et suiv.) qui distingue l'inscription sur la liste de sauvegarde, le patrimoine exceptionnel, le petit patrimoine populaire et le patrimoine exceptionnel. 
comme l'agence Onroerende Erfoed en Région flamande, jouent également un rôle d'appui, voire de contrôle, en matière de biens classés.

\section{4. ÉVALUATION : UN DISPOSITIF ÉQUITABLE?}

Comme évoqué précédemment, appréhender l'action publique relative au patrimoine religieux implique de prendre en compte plusieurs politiques publiques, à savoir - schématiquement - celle relative à ce que l'on appelait autrefois « la politique des cultes » et celle relative à la préservation du patrimoine historique.

À cet égard, les interventions publiques peuvent être jaugées à l'aune de l'équité, dont le respect est généralement considéré comme un élément de base du "vivre ensemble ", thème récurrent du discours politique. Cela amène à appréhender dans leur globalité les effets des deux grands axes de politiques publiques cités ci-dessus.

Un constat de départ est que les cultes et les organisations philosophiques sont inégalement dotés en ressources, notamment monétaires (par exemple les ressources propres), cognitives (par exemple la maîtrise des dossiers de subvention), interactives (par exemple un réseau d'appui ou de coordination), patrimoniales (infrastructure) ou majoritaires (soutien politique) ${ }^{33}$. Dès lors, la recherche d'une forme d'équité doit-elle viser à corriger cette dotation inégale? Si l'on se limite aux ressources monétaires et patrimoniales, force est de constater que le problème est multiforme.

D'abord, certains cultes, présents en Belgique depuis des siècles, disposent d'un certain patrimoine et de lieux de culte en suffisance, au contraire d'autres cultes, d'implantation plus récente. Dès lors, dans un régime de soutien public aux cultes et organisations philosophiques reconnus, convient-il d'organiser un "rattrapage " afin d'aider les nouveaux arrivés sur le "marché religieux » ? Cela n'a guère été le cas et les interventions pour la construction de nouveaux lieux de culte sont facultatives. À notre connaissance, il n'y a guère eu de subventions octroyées dans ce cadre à l'exception des Maisons de la laïcité, alors considérées comme l'ancrage local de l'assistance philosophique non confessionnelle ${ }^{34}$. Les cultes en développement, qui auraient

33. Pour une liste des divers types de ressources, V. Knoepfel P., Larrue C. et Varone F., Analyse et pilotage des politiques publiques, Zürich, Verlag Rüegger, $2^{\mathrm{e}}$ éd. 2006.

34. Husson J.-F., "Le financement des cultes, de la laïcité et des cours philosophiques», art. cit. ; HusSON J.-F. et SÄGESSER C., « La reconnaissance et le financement de la laïcité (II)», art. cit. ; Jeholet P.-Y., SÄGesser C. et Husson J.-F, op. cit. 
pu ou pourraient prétendre à un tel « rattrapage », n'en ont guère bénéficié. Cependant, plusieurs cas peuvent être mentionnés de bâtiments appartenant aux pouvoirs publics et mis à disposition, voire cédés pour un euro symbolique, afin d'en faire des mosquées ${ }^{35}$. Le cas échéant, cette absence de soutien public à de nouveaux lieux de culte renvoie au problème de la recherche de financements et, partant, pose la question des financements étrangers, avérés dans plusieurs cas.

Ensuite, certains cultes sont confrontés à un niveau de dépenses plus élevé, qu'il s'agisse du fonctionnement ${ }^{36}$ ou du coût des travaux de rénovation ou d'entretien. Sur ce point, les travaux sur des bâtiments classés doivent respecter des normes et procédures particulièrement rigoureuses, ce qui n'est pas sans impact budgétaire. D'autres cultes pourront compter sur une plus grande générosité des fidèles. La corrélation avec la dotation en capital cognitif peut également jouer, comme l'illustre le cas du culte catholique : fonctionnant - pour l'essentiel - sur la base des mêmes dispositions depuis plus de deux siècles, bénéficiant d'un réseau de fabriciens très actif, de services d'appui à la gestion des paroisses dans les évêchés et d'un monde associatif dynamique (quoique vieillissant), sa capacité à obtenir des financements tant publics que privés est davantage présente qu'au sein d'autres cultes.

Enfin, malgré les questions qu'il peut soulever, le dispositif existant présente un avantage par rapport aux situations française et anglaise. Dans ces deux cas - que l'on présente souvent comme étant aux antipodes en matière de régimes des cultes, entre République laïque et Église établie -, il n'y a pas de financement organique des cultes autrement que par la voie d'incitations fiscales en faveur des dons consentis. Le fonctionnement et l'entretien des lieux de cultes reposent donc sur la contribution des fidèles et la capacité d'obtenir d'autres ressources privées. Une exception réside alors dans le fait que les bâtiments classés peuvent obtenir des aides d'État ou d'organismes paraétatiques au titre du patrimoine historique. Or, seuls les cultes bénéficiant d'une certaine implantation historique peuvent en bénéficier et, de ce fait, tel n'est pas le cas du culte musulman en France ou en Angleterre, hormis de rares exceptions, générant un effet de renforcement de l'inégalité constatée.

35. Outre le cas de la Grande Mosquée de Bruxelles, déjà évoqué, citons la mosquée d’Andenne, cédée par la municipalité pour l'euro symbolique. Remarquons qu'aucune de ces mosquées n'est aujourd'hui reconnue par les pouvoirs publics.

36. Telles les églises catholiques, en général assez anciennes, grandes et énergivores. 
Outre l'équité, d'autres critères d'évaluation peuvent être pris en considération telles l'efficacité, l'efficience et la légitimité. Illustrons-les par des exemples significatifs, sans toutefois prétendre à une quelconque exhaustivité.

L'efficacité impliquerait que, d'une part, les cultes et organisations philosophiques non confessionnelles disposent de lieux de culte adéquats et, d'autre part, que les lieux de culte présentant un intérêt au sens de la préservation du patrimoine puissent être conservés correctement. L'appréciation peut être globalement positive à l'égard de ces deux arguments.

À l'égard du premier argument, relevons toutefois que certaines communautés cultuelles ne disposent pas de bâtiments adéquats (problèmes de taille, de sécurité...) et que d'autres en disposent, grâce à certains financements étrangers (en particulier des États du Golfe). Ces deux types de situations sont intimement liés à la question de la reconnaissance des communautés cultuelles locales. Souvent, les communautés concernées ne souhaitent pas être reconnues, afin d'éviter les contraintes administratives liées à un tel statut ; d'autres ne remplissent pas les conditions fixées pour être reconnues ; d'autres encore ne trouvent guère d'intérêt à une telle reconnaissance, s'autofinançant auprès des fidèles et de donateurs étrangers, ces derniers pouvant promouvoir des positionnements idéologiques polarisants. Cette dernière situation va à l'encontre du « vivre ensemble » évoqué précédemment.

À l'égard du second argument, force est de constater que les institutions en charge du patrimoine sont parfois amenées à établir des priorités et qu'il n'est pas possible de "préserver tout». Le sort des églises construites à la fin du XIX ${ }^{e}$ siècle illustre ce débat à propos de ce qu'il est opportun de maintenir et de sauvegarder ou pas.

L'efficience, entendue comme une minimisation des coûts pour un niveau fixé de production publique ou une production publique maximale pour un niveau de coûts donné, peut également être appréciée positivement. Les pistes pour améliorer globalement l'efficience ont, ces dernières années, été orientées dans plusieurs directions. Une de celles-ci consiste en la rationalisation des acteurs publics en présence, comme en témoigne l'annonce récente d'un regroupement au sein d'un même organisme des deux principales institutions wallonnes compétentes en la matière ${ }^{37}$. Une autre piste porte sur la réaffectation des lieux de culte. À cet égard, l'analyse coûts-bénéfice doit

37. Le ministre compétent a annoncé en septembre 2016 le regroupement de la Division du patrimoine du service public de Wallonie et de l'Institut du patrimoine wallon au sein d'un nouvel organisme : http://prevot.wallonie.be/un-interlocuteur-unique-pour-le-patrimoineen-wallonie-plut-t-que-deux-structures-en-comp-tition [consulté le 13 janv. 2017]. 
cependant être approfondie, car une réaffectation, le plus souvent à un but culturel, requiert des moyens budgétaires substantiels ${ }^{38}$ même si elle permet d'économiser le coût de construction d'un bâtiment neuf.

Enfin, en matière de légitimité, les réactions du public sont généralement contradictoires. D'une part, on observe une réticence à financer des églises peu fréquentées ou les lieux de culte des religions minoritaires. D'autre part, une demande fortement imprégnée de consumérisme religieux va se traduire par l'exigence de pouvoir disposer d'un lieu de culte proche pour certaines cérémonies marquantes ${ }^{39}$. Dans le même ordre d'idées, peuvent être relevés plusieurs cas d'opposition à la réaffectation d'un lieu de culte ou, a contrario, de soutien à des projets de restauration des bâtiments cultuels classés considérés comme étant d'importance majeure.

\section{LE FUTUR : ADAPTATION, RÉFORME OU RÉVOLUTION ?}

Nombre d'acteurs, tant du côté des cultes que des pouvoirs publics - en particulier locaux - considèrent que des efforts doivent être réalisés afin de réduire les charges des pouvoirs locaux. Si ces dernières sont relativement limitées - de l'ordre d'un pour cent des dépenses ordinaires -, ces interventions, longtemps relativement préservées, ont été remises en cause au fur et à mesure des difficultés budgétaires des pouvoirs locaux. Les autorités catholiques en sont d'ailleurs conscientes et, dans plusieurs évêchés, des réflexions et démarches ont été entamées afin de permettre certaines rationalisations.

Confrontée à l'existence d'un parc immobilier supérieur à ses besoins d'assistance religieuse, l'Église catholique a recours à plusieurs pistes, au cas par cas. Une des pistes privilégiées est le transfert à une autre communauté catholique, par exemple d'origine immigrée ou encore charismatique ${ }^{40}$. Une autre piste est le transfert vers d'autres communautés chrétiennes, en

38. Pour plus de détails, V. notamment Hennart E., "Patrimoine et réaffectation : le rôle de l'Institut du patrimoine wallon », in Husson J.-F. (éd.), Le financement des cultes et de la laïcité. Comparaison internationale et perspectives, Namur, Éd. namuroises, 2005, p. 157-168; Compte rendu intégral de la Commission des travaux publics, de l'agriculture, de la ruralité et du patrimoine du Parlement de Wallonie, séance du jeudi 26 avril 2012 : http://nautilus.parlement-wallon.be/Archives/2011_2012/CRIC/cric121.pdf [consulté le 13 janv. 2017].

39. Tels les baptêmes, mariages et funérailles dans le cas du culte catholique.

40. Ces derniers cas ont régulièrement fait l'objet de réserves, voire d'oppositions, des paroissiens voire des fabriciens. 
particulier orthodoxes ${ }^{41}$. Parfois, sans que la propriété soit modifiée, des églises catholiques accueillent des célébrations d'autres cultes chrétiens ${ }^{42}$.

Une piste, tantôt privilégiée, tantôt considérée avec méfiance dans les milieux catholiques, est celle de l'utilisation partagée entre une affectation cultuelle et un usage culturel. L'utilisation partagée vise à préserver le culte, tout en ouvrant l'église à diverses activités culturelles. Outre des arrangements ponctuels entre la fabrique et la commune, cela peut prendre un aspect formel comme dans le cadre de la convention relative à la chapelle Notre-Dame-du-Marché, à Jodoigne, apparemment première du genre. Cette convention, conclue entre la Province du Brabant wallon, la ville de Jodoigne, la fabrique d'église et l'Institut du patrimoine wallon, détaille les modalités d'organisation d'activités touristiques et culturelles s'ajoutant aux activités cultuelles et permet des modalités de financement ad hoc ${ }^{43}$. Si un tel dispositif réduit quelque peu la marge de manœuvre cultuelle, elle permet par contre de bénéficier de canaux de financement supplémentaires, généralement dans le cadre des budgets culturels relevant des pouvoirs locaux ou des autorités supérieures.

Reste la piste de la désaffectation suivie, le cas échéant, de la vente du lieu de culte, imposant une procédure assez lourde que les acteurs souhaitent voir simplifiée ${ }^{44}$. Plusieurs églises ont ainsi été reconverties en maison de quartier ou de village, en bibliothèque ou en centre culturel. De telles reconversions nécessitent toutefois des moyens importants pour l'aménagement des lieux, voire pour le fonctionnement ${ }^{45}$. Parfois, l'église désaffectée rejoint le giron du secteur privé pour devenir un hôtel-restaurant ou un espace commercial.

41. Il y a parfois des situations tout à fait particulières, comme une église mise provisoirement à disposition d'une communauté musulmane dont la mosquée avait été détruite. Il arrive aussi que des églises mises en vente soient acquises par des communautés " indépendantes », telle celle constituée autour de l'ancien prêtre catholique se faisant appeler Père Samuel, lequel a notamment été cité lors des travaux de la commission parlementaire sur les sectes (Enquête parlementaire visant à élaborer une politique en vue de lutter contre les pratiques illégales des sectes et le danger qu'elles représentent pour la société et pour les personnes, particulièrement les mineurs d'àge. Rapport fait au nom de la commission d'enquête par MM. Duquesne et Willems, Doc. Parl. Ch., 313 / 8 - 95 / 96 , p. $160,175,262$ ).

42. Cela s'accompagne parfois de tensions liées à l'aménagement des lieux, à l'entretien et aux coûts.

43. Pour plus de détails, V. notamment le compte rendu intégral de la Commission des travaux publics, de l'agriculture, de la ruralité et du patrimoine du Parlement de Wallonie, séance du jeudi 26 avril 2012, précit.

44. La lourdeur découle de l'existence, dans plusieurs Régions, d'une double tutelle religieuse et administrative.

45. V. source citée dans la note 43. 
Cette dernière affectation suscite généralement des réserves, a fortiori lorsque des éléments de l'ancienne affectation cultuelle sont conservés.

Enfin, depuis le début des années 2000, des débats relatifs à une refonte globale du système de financement ont régulièrement émergé, sans grande conséquence à ce jour. Compte tenu de l'éclatement des compétences entre autorité fédérale et entités fédérées, une révolution en la matière semble difficile. Ainsi, le recours à un impôt philosophiquement dédicacé ${ }^{46}$ pourrait difficilement s'étendre au-delà des compétences fédérales, c'est-à-dire des traitements des ministres des cultes et délégués des organisations philosophiques. Par contre, les Régions pourraient décider de revoir les modalités de financement par leurs pouvoirs locaux. Une piste formulée, s'inspirant des développements récents au Grand-duché de Luxembourg ${ }^{47}$, consisterait à regrouper les biens immobiliers dans une fondation qui, au sein de chaque culte, gérerait l'ensemble des biens immobiliers. Si pareille organisation pourrait - vraisemblablement non sans mal - être mise en place du côté catholique, cela apparaît impossible au sein des cultes protestant-évangélique ou musulman, au sein desquels une telle gestion centralisée se heurterait à la diversité interne. Une autre piste pourrait limiter les interventions aux seuls bâtiments, les autres dépenses devant être supportées par les fidèles ${ }^{48}$. Un tel développement n'apparaît guère envisageable actuellement, car, comme mentionné précédemment, cela amènerait, sur un même territoire, à ce que les organisations philosophiques bénéficient d'un financement plus généreux que les cultes ${ }^{49}$.

\section{REMARQUES CONCLUSIVES}

Le cadre légal et réglementaire belge relatif aux lieux de culte reste fortement basé sur des instruments de régulation et de financement remontant au début du XIX ${ }^{e}$ siècle, même s'il a cependant pu s'ouvrir à de nouveaux courants religieux et philosophiques.

La situation est toutefois devenue d'une grande complexité, car les producteurs de politiques publiques doivent tenir compte de la régionalisation des

46. S'inspirant, par exemple, du huit pour mille italien.

47. V. Jeholet P.-Y., Sägesser C. et Husson J.-F., op. cit., p. 57. - V. aussi chronique de F. Messner dans ce numéro.

48. Hormis le traitement du desservant qui resterait à charge de l'autorité fédérale, sauf changement à ce niveau.

49. Pour un relevé commenté de ces scénarios: Jeholet P.-Y., Sägesser C. et Husson J.-F, op. cit. 
compétences liées aux cultes et au patrimoine immobilier classé, amenant à des cadres différents; de communautés convictionnelles dont les niveaux de pratiques, les ressources monétaires et de patrimoine diffèrent grandement ; des interrelations entre la politique à l'égard des cultes et organisations philosophiques d'une part, et les autres politiques - dont celle du patrimoine classé - d'autre part.

Les difficultés rencontrées par les pouvoirs locaux exercent une pression sur l'ensemble des pouvoirs publics ainsi que sur les autorités catholiques pour aller vers une rationalisation du système. Parallèlement, se pose la question des modalités d'un soutien aux lieux de culte pour les cultes en croissance, en l'absence duquel ces derniers devraient trouver ailleurs les moyens nécessaires, soulevant dès lors la délicate question des financements étrangers - particulièrement sensible dans le cas de l'islam.

Enfin, toute réforme en matière de relations entre les pouvoirs publics et les cultes et organisations philosophiques, fut-elle limitée au mode de financement, se devra de prendre en compte la question du patrimoine immobilier, classé ou non. 